\title{
Beyond Glutamate Antagonists for Treatment of Ischemic Stroke: Blood Glutamate Grabbing
}

\author{
José Castillo', María Isabel Loza ${ }^{2,3}$, David Mirelman ${ }^{4}$, Tomás Sobrino', Francisco Campos' \\ 'Department of Neurology, Clinical University Hospital, Universidade de Santiago de Compostela, Health Research Institute of Santiago de Compostela, Spain. \\ ${ }^{2}$ Department of Pharmacology, Universidade de Santiago de Compostela, Discovery group BioFarma, Health Research Institute of Santiago de Compostela, Spain. \\ ${ }^{3}$ BioFarma Research Group, Centro de Investigación en Medicina Molecular y Enfermedades Crónicas (CIMUS), University of Santiago de Compostela, Santiago \\ de Compostela, Spain. \\ ${ }^{4}$ Department of Biological Chemistry, Weizmann Institute of Science, Rehovot, Israel.
}

Article Info

\section{Article Notes}

Received: May 18, 2016

Accepted: June 21, 2016

\section{*Correspondence:}

Francisco Campos

Servicio de Neurología

Hospital Clínico Universitario

Travesa da Choupana s $/ \mathrm{n}$

15706 Santiago de Compostela

Spain

E-mail: jose.castillo.sanchez@sergas.es

francisco.campos.perez@sergas.es

(c) 2016 Castillo J. This article is distributed under the terms of the Creative Commons Attribution 4.0 International License

\section{Keywords}

Blood brain barrier

Blood glutamate grabbing

Glutamate excitotoxicity

Neuroprotection

Cerebra Ischemia

\section{ABSTRACT}

Glutamate-excitotoxicity is a primary contributor of ischemic neuronal death. Several strategies have been developed against glutamate-excitotoxicity, however any of them have not showed positive results in the clinical practice so far.

Nowadays, the concept of blood/brain glutamate grabbing is well recognized as a novel and attractive protective strategy to reduce the excitotoxic effect of excess extracellular glutamate that accumulates in the brain following an ischemic stroke. The main advantage of this novel therapeutic strategy is that occurs in the blood circulation and therefore does not affect the normal brain neurophysiology, as it has been described for other drug treatments used against glutamate excitotoxicity. In this work, we summarize all experimental data about the potential application of this therapy against stroke pathology.

Ischemic stroke, caused by interruption of the blood supply to the brain, is one of the most important causes of morbidity and mortality worldwide. Currently, the control of systemic parameters, such as body temperature, blood pressure, and glycemia, has considerably improved the outcome of stroke patients. In the absence of protective therapy, an early artery reperfusion, i.e. mechanical or enzymatic thrombolysis, remains the primary goal of treatment for acute ischemic stroke. However, because of the progressive increase in stroke incidence, the high morbidity and the limited therapeutic tools against stroke, seeking new alternatives that can be applied more universally and with less technological requirements is in high demand $^{1,2}$

\section{Glutamate excitotoxicity in Stroke}

It is well known that glutamate-mediated excitotoxicity is a primary contributor to ischemic neuronal death. Glutamate excitotoxicity appears when the neurotransmitter's homeostatic balance is disrupted and levels become elevated in the extracellular fluid $^{3}$. Whereas extracellular glutamate levels are increased during neuronal damage, in normal physiological conditions, high concentrations of neurotransmitters in the brain are stored intracellularly. The concentration of extracellular glutamate is tightly regulated to maintain physiological concentrations through sodium-dependent transporters (also known as Excitatory Amino 
Acid Transporters; EAATs). Reuptake of glutamate from synaptic junctions after neuron excitation normally involves the participation of EAATs transporters located on astrocytes, which bind and remove the neurotransmitter for processing and recycling. Alternatively, when extracellular concentrations become elevated, sodium-dependent transport located on the antiluminal surface of brain capillary endothelial cells are able to transfer glutamate from the extracellular space. When glutamate accumulates in the endothelial cells to a concentration that exceeds plasma levels, it is moved via facilitated diffusion through the luminal side into the blood stream. In this regard, the endothelial regulation of glutamate concentration can occur despite unfavorable concentration gradients from brain to plasma ${ }^{4,5}$.

The failure of nutrient supply after ischemia causes a neuronal depolarization leading to a massive release of glutamate to the extracellular space. In addition, due to glutamate uptake is a high energy-dependent process, this restriction of energy causes a drastic disruption of the glutamate transporters enhancing the excitotoxic effect to trigger the death of neurons ${ }^{6,7}$.

More than 15 years ago, we reported, in clinical studies, that glutamate is critical for neuronal damage after ischemic stroke ${ }^{8-10}$. Ischemic patients presented higher blood and Cerebrospinal fluid (CSF) glutamate levels than control subjects at admission ${ }^{8,9}$, suggesting that glutamate concentrations above $200 \mu \mathrm{M}$ in plasma acted as an important predictor of neuronal damage at 48 hours, with a sensitivity of $85 \%$ and a specificity of $97 \%{ }^{11}$. Moreover, high levels of glutamate in the plasma for at least 24 hours was associated with early neurological deterioration, whereas in patients with stable ischemic stroke, glutamate levels dropped to normal values less than 6 hours from onse $^{11}$. All of these clinical data demonstrated for the first time the critical role of glutamate in stroke pathology and suggested a source for new strategies for the research of new protective therapies based on the inhibition of glutamate toxicity. Therefore, controlling the increase of extracellular glutamate may confer neuroprotection by terminating multiple downstream death signaling cascades at their converging upstream initiation point.

Knowledge of the molecular mechanisms involved in glutamate excitotoxicity after cerebral ischemia allowed researchers to develop promising pharmacological strategies against this neurotoxic process. In the beginning, the major focus of research centered on NMDA receptors (NMDAr) antagonism. NMDAr provided a logical target for drug design because it represented a major gateway for the myriad of other downstream effects of glutamate excitotoxicity ${ }^{7}$.

Several classes of NMDAr antagonists with different sites of action were developed. Though showing promise in animal studies, antagonist drugs have largely failed in randomized, controlled clinical trials in humans. A variety of reasons have been postulated to explain the lack of success for these NMDAr-targeting therapies. Many of these compounds lack sufficient brain penetrance while exhibiting significant dose-limiting side effects. And others caused adverse events as hallucinations, agitations, catatonia, peripheral sensory loss, nausea, and elevation in blood pressure ${ }^{7}$.

\section{Blood to Brain Glutamate Grabbing}

High glutamate concentrations at the synaptic cleft are rapidly reduced by the action of glutamate transporters present primarily on astrocytes surrounding the nerve terminal to prevent glutamate excitotoxicity. In addition to astrocytes, EAATs, on the antiluminal membrane act to accumulate the excess extracellular glutamate into the endothelial cells. When the endothelial glutamate concentration becomes higher than the blood glutamate concentration, glutamate is transported into the blood by means of facilitated diffusion, a mechanism that facilitates blood excretion of glutamate from the brain ${ }^{4,5,7}$. Based on this mechanism, a decrease of blood glutamate levels by means of glutamate scavengers or grabbers leads to a larger glutamate gradient between the brain and blood, facilitating the lowering of extracellular glutamate in the brain, and reducing the toxic effects of this neurotransmitter. Therefore, manipulating this mechanism may have potential neuroprotective effects after stroke ${ }^{5,12}$.

To demonstrate this glutamate grabbing hypothesis, the blood resident enzyme glutamate-oxaloacetate transaminase (GOT), which transforms glutamate into $\alpha$-ketoglutarate and aspartate in the presence of oxaloacetate (OxAc), was used. This enzyme, when OxAc is artificially increased shifts the equilibrium of the reaction to the right side, thereby decreasing glutamate levels in blood $^{5,12}$.

The first evidence of the neuroprotective effect with OxAc in cerebral ischemia was observed in rats submitted to photothrombotic lesions ${ }^{13}$. This effect was subsequently probed in a model of ischemia induced by the transient occlusion of the middle cerebral artery (MCAO) ${ }^{14}$. Under the STAIR guidelines OxAc was provided $90 \mathrm{~min}$ after occlusion, leading to decreased blood glutamate levels, following by a decrease in infarct volume and edema after ischemia. These effects were associated with a reduction in motor deficit. To confirm that the neuroprotective effect was due to a decrease in brain glutamate levels, spectroscopic analysis revealed that the increase in brain glutamate observed in control animals after MCAO was clearly reduced in animals treated with OxAc. These results were also validated by other independent laboratories ${ }^{15}$. 
To further demonstrate the clinical relevance of these pre-clinical results as potential therapeutic strategies, high blood levels of GOT was later hypothesized to be correlated with lower blood glutamate levels and subsequently with a better functional outcome. To test this hypothesis, two independent clinical and observational studies were performed, in which the primary end point was functional outcome at 3 months ${ }^{16,17}$. In these studies, patients with good outcomes showed lower glutamate levels and higher GOT levels in blood samples collected at admission. A significant inverse correlation was observed between GOT and glutamate levels. The favorable effect on functional outcome was also supported by reduced lesion volumes. These clinical findings represented the first clinical evidence of the neuroprotective effect of blood glutamate grabbing mechanisms in ischemic stroke patients and supported the potential applicability of OxAc or GOT as future treatments for acute ischemic stroke.

Indeed, human recombinant GOT1 (rGOT1) was later tested in the rat model of ischemia induced by transient MCAO and it was observed a reduction in serum and brain glutamate levels, resulting in a reduction in infarct volume and sensorimotor deficit ${ }^{18}$.

\section{Repositioning of Drugs against Glutamate Toxicity}

All preclinical studies on different models and the clinical observational analysis reported guarantee the therapeutic efficacy of the reduction of blood glutamate as well as the glutamate grabber drugs; however, translation to clinical practice has critical steps before their use in humans. These critical steps are those necessary to develop a clinical trial, which will involve high financial support and risk of investment for the sponsors interested in the study. In addition, the repeated failure of protective drugs against glutamate excitotoxicity in clinical trials has reduced the trust of pharmaceutical companies and other sponsors in stroke studies.

Aiming to demonstrate the clinical efficacy glutamate grabbers in humans and reduce the risk of investment in the study, the pharmacological strategy known as drug repositioning represents an interesting alternative to find new grabbing drugs ${ }^{19,20}$.This allowed researchers to search drugs already known and used for other pathologies and in which the clinical phase I and phase II were already completed, reducing the risk of investment for sponsors in case the clinical study result failed.

Following this pharmacological strategy, a new clinical study (proof of concept) with a new grabbing drug is currently ongoing (EudraCT Number: 2014-003123-22).

In conclusion, the critical role of glutamate excitotoxicity has been long described as a key molecular cause of neuronal injury from stroke. At present, we have a better understanding of the mechanisms by which elevated glutamate ultimately leads to cell death; unfortunately all clinical trials that have been primarily based on the inhibition of glutamate excitotoxicity through glutamate antagonist to date have failed. Currently, many experimental evidences in different models of diseases use blood glutamate grabbers as effective treatments against neuronal damage induced by glutamate excitotoxicity. Future clinical trials (some of them in progress) will allow to know the application of this novel therapeutic strategy.

\section{Acknowledgements}

This study has been partially supported by grants from Instituto de Salud Carlos III (PI13/00292; PI14/01879). T. Sobrino (CP12/03121) and F. Campos (CP14/00154) are recipients of a research contract from Miguel Servet Program of Instituto de Salud Carlos III. The funders had no role in the study design, data collection and analysis, decision to publish, or preparation of the manuscript.

\section{References}

1. Howard G, Goff DC. Population shifts and the future of stroke: Forecasts of the future burden of stroke. Ann N Y Acad Sci. 2012;1268:14-20

2. Krishnamurthi RV, Feigin VL, Forouzanfar MH, et al. Global and regional burden of first-ever ischaemic and haemorrhagic stroke during 19902010: Findings from the global burden of disease study 2010. Lancet Glob Health. 2013;1:e259-281

3. Ramos-Cabrer P, Hoehn M. Mri stem cell tracking for therapy in experimental cerebral ischemia. Transl Stroke Res. 2012;3:22-35

4. O'Kane RL, Martinez-Lopez I, DeJoseph MR, Vina JR, Hawkins RA. $\mathrm{Na}(+)$-dependent glutamate transporters (eaat1, eaat2, and eaat3) of the blood-brain barrier. A mechanism for glutamate removal. J Biol Chem. 1999;274:31891-31895

5. Teichberg VI, Cohen-Kashi-Malina K, Cooper I, Zlotnik A. Homeostasis of glutamate in brain fluids: An accelerated brain-to-blood efflux of excess glutamate is produced by blood glutamate scavenging and offers protection from neuropathologies. Neuroscience. 2009;158:301-308

6. Danbolt NC. Glutamate uptake. Prog Neurobiol. 2001;65:1-105

7. Jia M, Njapo SA, Rastogi V, Hedna VS. Taming glutamate excitotoxicity: Strategic pathway modulation for neuroprotection. CNS Drugs. 2015;29:153-162

8. Castillo J, Davalos A, Naveiro J, Noya M. Neuroexcitatory amino acids and their relation to infarct size and neurological deficit in ischemic stroke. Stroke. 1996;27:1060-1065

9. Castillo J, Davalos A, Noya M. Progression of ischaemic stroke and excitotoxic aminoacids. Lancet. 1997;349:79-83

10.Castillo J, Martinez F, Corredera E, Aldrey JM, Noya M. Amino acid transmitters in patients with headache during the acute phase of cerebrovascular ischemic disease. Stroke. 1995;26:2035-2039

11. Castellanos M, Sobrino T, Pedraza S, et al. High plasma glutamate concentrations are associated with infarct growth in acute ischemic stroke. Neurology. 2008;71:1862-1868

12. Campos F, Sobrino T, Ramos-Cabrer P, Castillo J. Oxaloacetate: A novel neuroprotective for acute ischemic stroke. Int J Biochem Cell Biol. 2012;44:262-265

13. Boyko M, Gruenbaum SE, Gruenbaum BF, Shapira Y, Zlotnik A. Brain to blood glutamate scavenging as a novel therapeutic modality: A review. J Neural Transm. 2014;121:971-979 
14. Campos F, Sobrino T, Ramos-Cabrer P, et al. Neuroprotection by glutamate oxaloacetate transaminase in ischemic stroke: An experimental study. J Cereb Blood Flow Metab. 2011;31:1378-1386

15. Knapp L, Gellert L, Kocsis K, Kis Z, Farkas T, Vecsei L, Toldi J. Neuroprotective effect of oxaloacetate in a focal brain ischemic model in the rat. Cell Mol Neurobiol. 2014

16. Campos F, Rodriguez-Yanez M, Castellanos M, et al. Blood levels of glutamate oxaloacetate transaminase are more strongly associated with good outcome in acute ischaemic stroke than glutamate pyruvate transaminase levels. Clin Sci (Lond). 2011;121:11-17

17. Campos F, Sobrino T, Ramos-Cabrer P, et al. High blood glutamate oxaloacetate transaminase levels are associated with good functional outcome in acute ischemic stroke. J Cereb Blood Flow Metab. 2011;31:1387-1393

18. Perez-Mato M, Ramos-Cabrer P, Sobrino T, et al. Human recombinant glutamate oxaloacetate transaminase 1 (got1) supplemented with oxaloacetate induces a protective effect after cerebral ischemia. Cell Death Dis. 2014;5:e992

19.Fagan SC. Drug repurposing for drug development in stroke. Pharmacotherapy. 2010;30:51S-54S

20. Corbett A, Pickett J, Burns A, et al. Drug repositioning for alzheimer's disease. Nat Rev Drug Discov. 2012;11:833-846 